\title{
Evaporative Drying of Sodium Chloride Solution Droplet on a Thermally Controlled Substrate
}

\section{Nandita Basu and Rabibrata Mukherjee*}

Instability and Soft Patterning Laboratory, Department of Chemical Engineering, Indian Institute of Technology Kharagpur, Pin 721 302, West Bengal, India

*Author for correspondence. E-mail id: rabibrata@che.iitkgp.ac.in

\section{Online Supporting Information}

\section{ROOM TEMPERATURE STUDY}

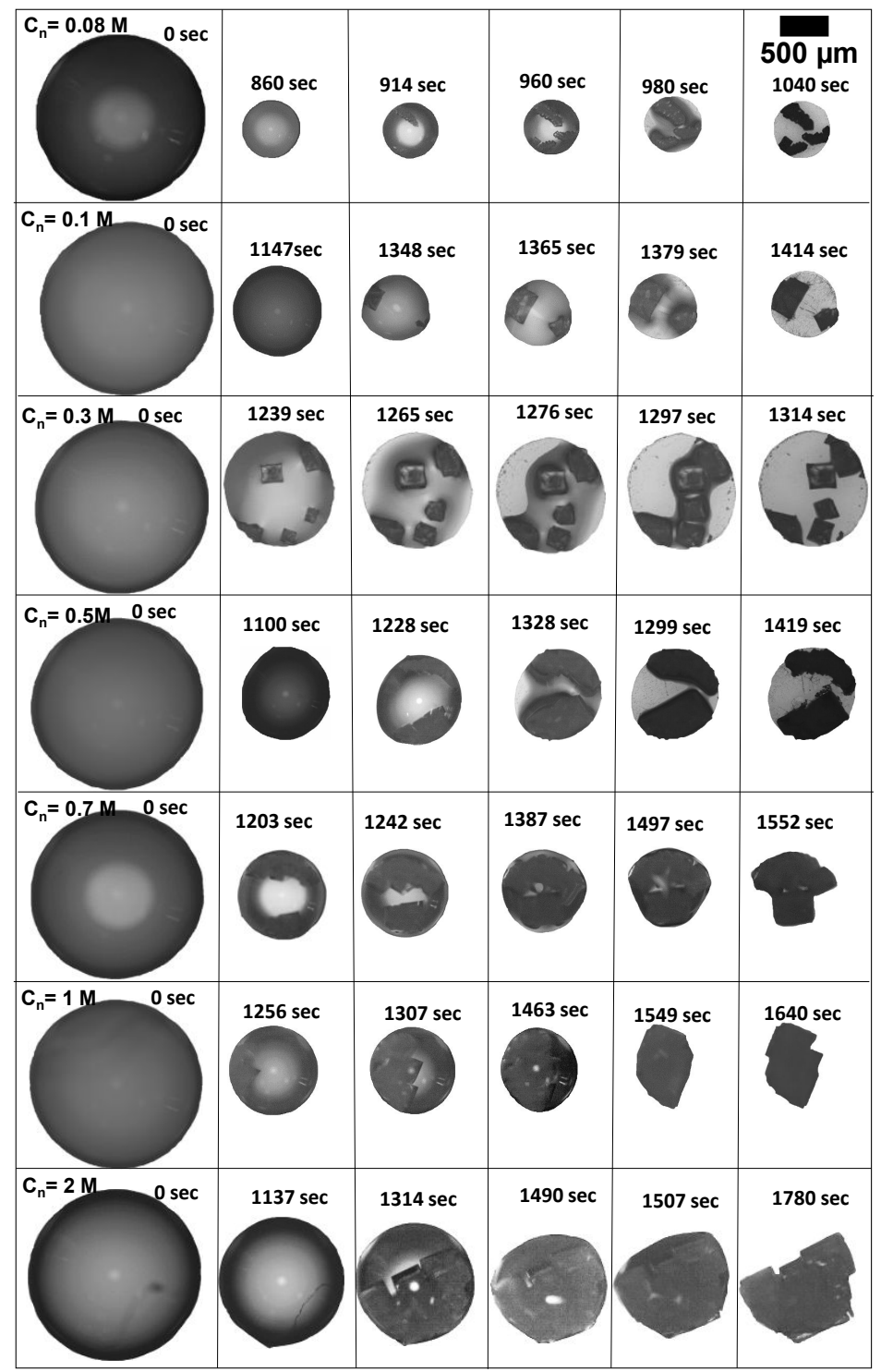

Figure S1: Real-time evaporative drying processes of $\mathrm{NaCl}$ solution droplets of various $C_{n}$ and final crystal morphologies at room temperature on flat PDMS. 


\section{At $40^{\circ} \mathrm{C}$}

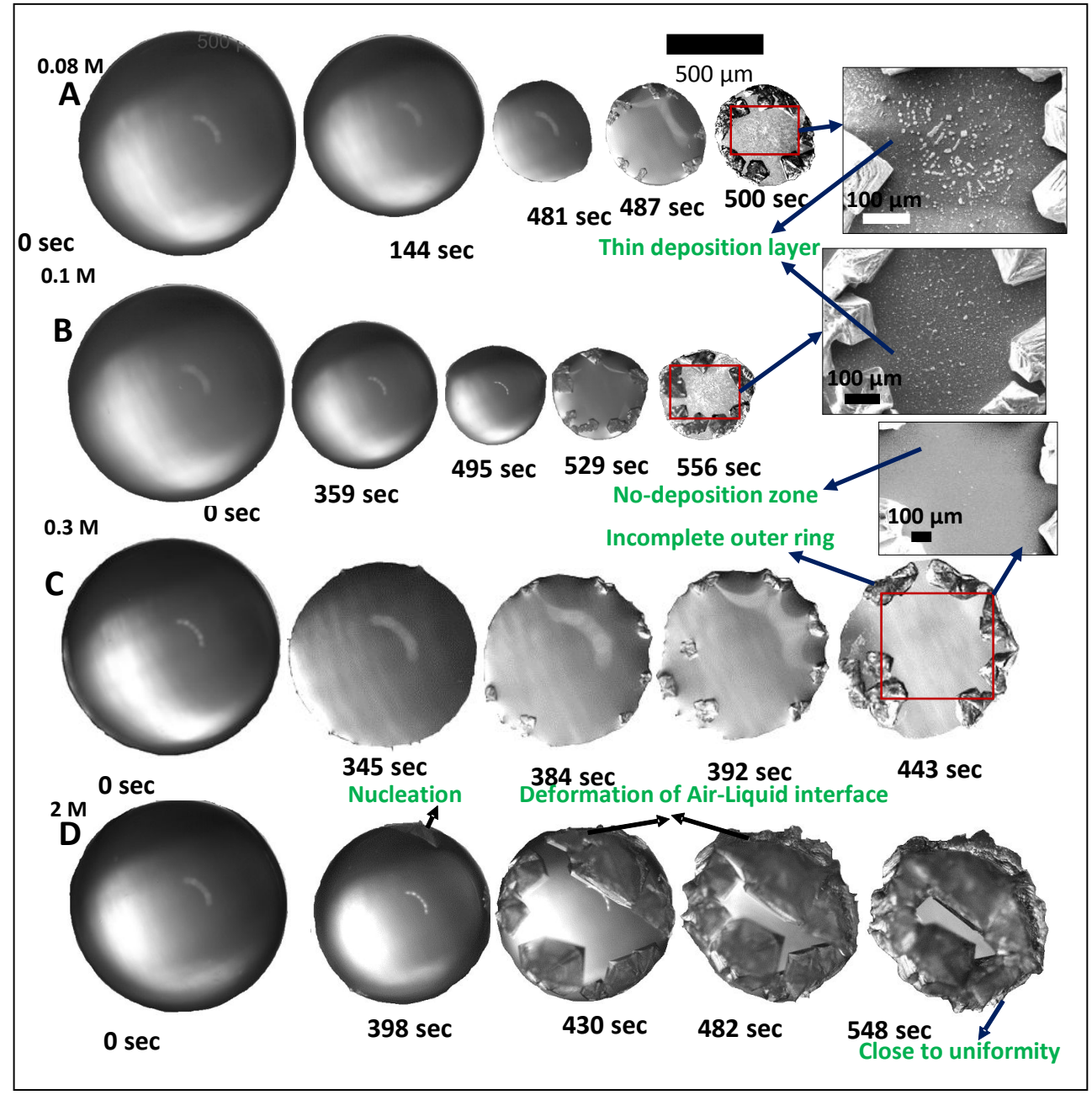

Figure S2: Real-time evaporative drying processes of $\mathrm{NaCl}$ solution droplets of various $C_{n}$ and final crystal morphologies at $40^{\circ} \mathrm{C}$ on flat PDMS. 


\section{At $5^{\circ} \mathrm{C}$}

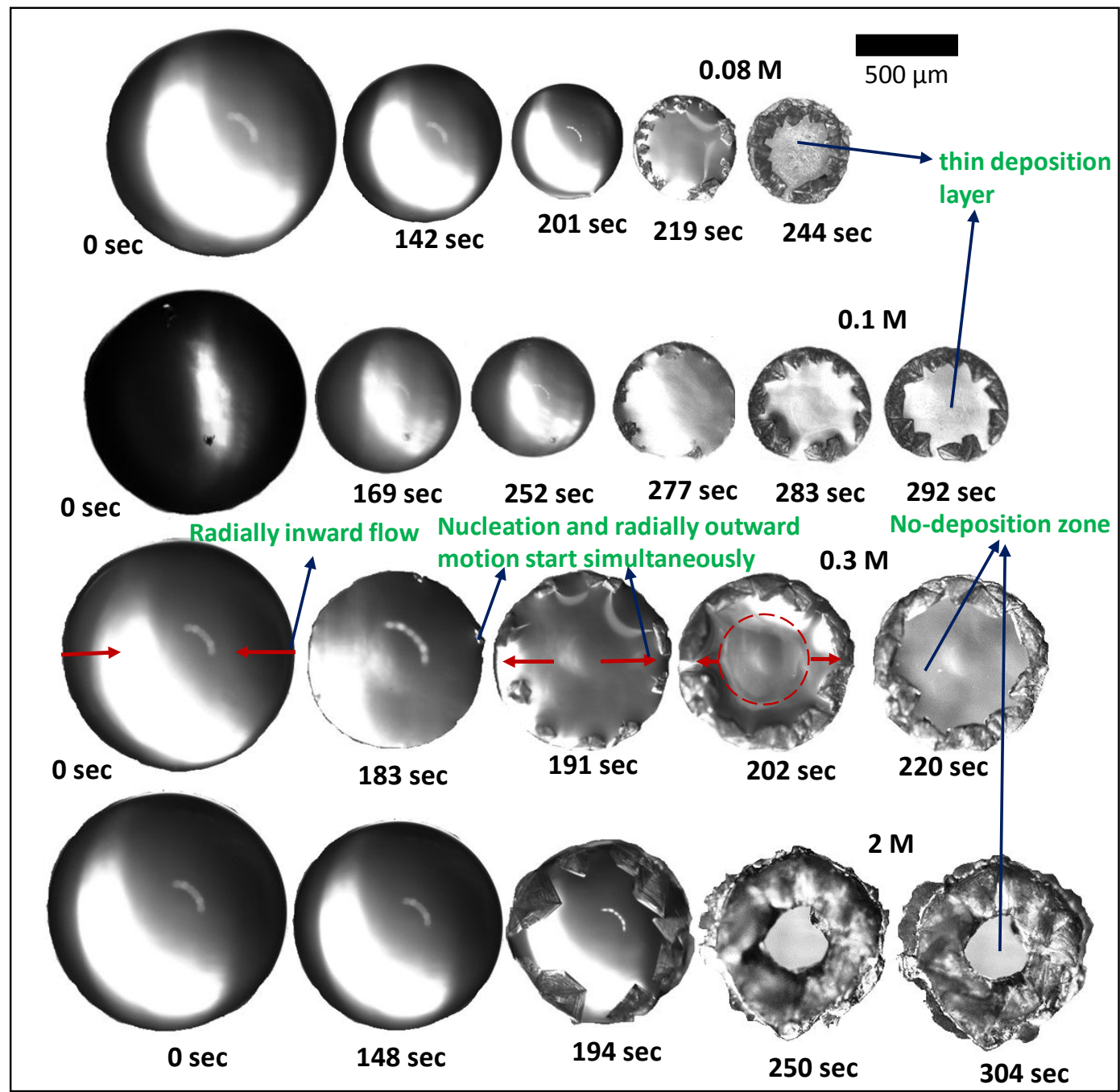

Figure S3: Real-time evaporative drying processes of $\mathrm{NaCl}$ solution droplets of various $C_{n}$ and final crystal morphologies at $55^{\circ} \mathrm{C}$ on flat PDMS. 

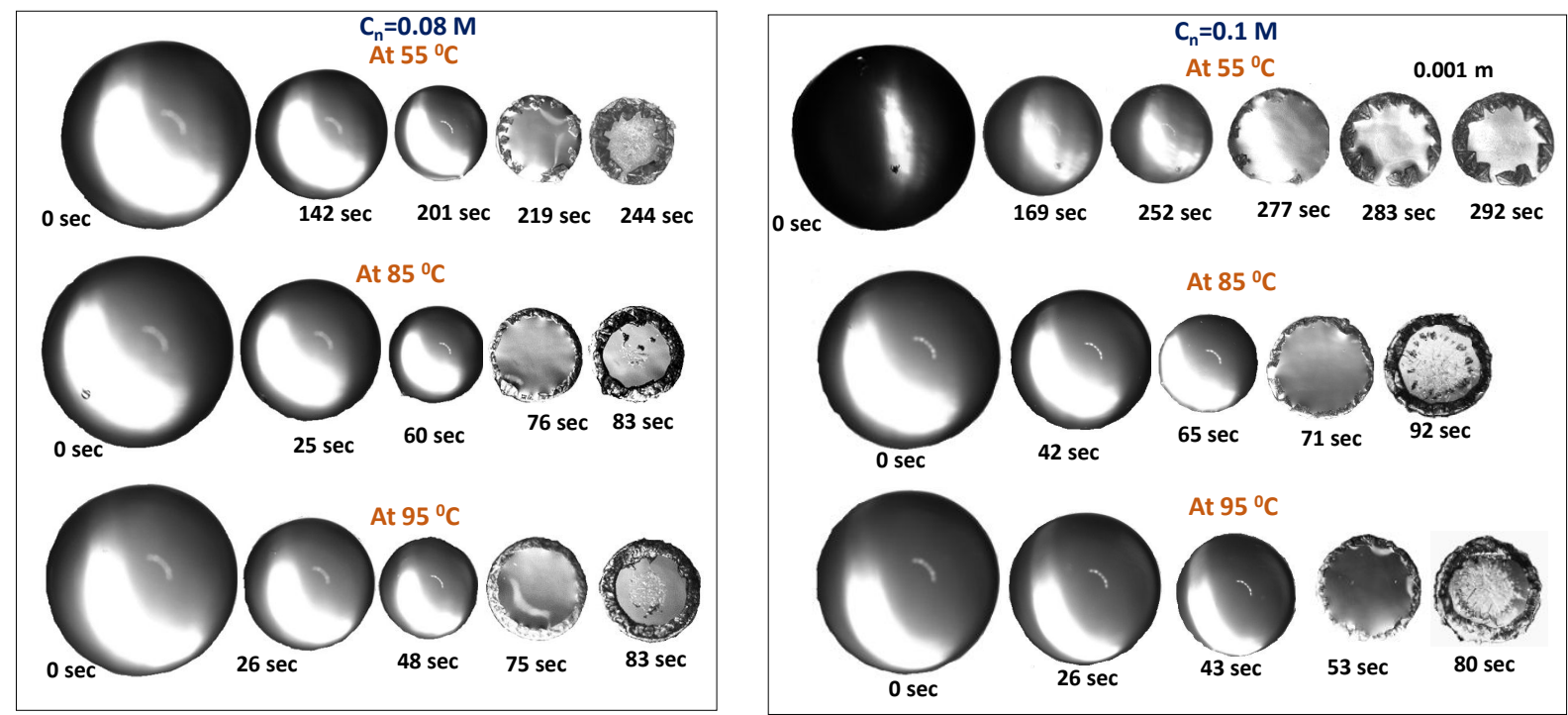

Figure S4: Real-time evaporative drying processes of $\mathrm{NaCl}$ solution droplets and final crystal morphologies at $55^{\circ} \mathrm{C}, 85^{\circ} \mathrm{C}$ and $95^{\circ} \mathrm{C}$ for $\mathrm{C}_{n}=0.08 \mathrm{M}$ and $0.1 \mathrm{M}$ of $\mathrm{NaCl}$ respectively on flat PDMS. 


\section{S5: Scaling of Marangoni flow and comparison with Capillary flow strength}

For a droplet with radius $\boldsymbol{R}$, the tangential stress arising from surface tension gradient, $\frac{\partial \gamma}{\partial \boldsymbol{X}}=\frac{\boldsymbol{\beta} \Delta \boldsymbol{T}}{\boldsymbol{R}}$ is balanced with the viscous stress $\frac{\eta v_{M}}{\boldsymbol{h}}$, where, $\eta$ is the liquid viscosity and $\boldsymbol{h}$ is the liquid film thickness. So, the Marangoni velocity $\left(\boldsymbol{v}_{\boldsymbol{M}}\right)$ scales as $\frac{\boldsymbol{\beta} \Delta \boldsymbol{T} \boldsymbol{h}}{\boldsymbol{\eta} \boldsymbol{R}}$. Liquid with contact angle $\boldsymbol{\theta}$ and thermal expansion $\boldsymbol{\alpha}=\frac{\partial \boldsymbol{\rho}}{\partial \boldsymbol{T}}$, Buoyancy $(\boldsymbol{\alpha} \Delta \boldsymbol{T} \boldsymbol{g} \boldsymbol{h} \boldsymbol{\theta})$ can be balanced with viscous stress $\frac{\boldsymbol{\eta} v_{B}}{\boldsymbol{h}}$ and the Buoyancy velocity $\left(\boldsymbol{v}_{\boldsymbol{B}}\right)$ scales as $\frac{\boldsymbol{\alpha} \Delta \boldsymbol{T} \boldsymbol{g} \boldsymbol{\theta} \boldsymbol{h}^{2}}{\eta} \cdot \frac{\boldsymbol{v}_{M}}{v_{B}}$ is independent of temperature difference and found to be $\frac{\boldsymbol{\beta}}{(\boldsymbol{\alpha} \boldsymbol{g h \boldsymbol { R } \theta})}$, a quantity which is always greater than 1 at the beginning of evaporation and increases with time as the liquid film thickness and contact angle decreases with evaporation. ${ }^{\mathbf{1 , 2}}$ This Marangoni flows also dominates Gravity guided flow as was confirmed by conducting the evaporative drying experiment turning the substrate upside down which results in similar evaporative morphology.

It has been discussed earlier that duration of capillary flow which is mainly responsible for the thick peripheral ring formation is significantly weaker than the Marangoni flow in our system. If the evaporation flux $\boldsymbol{j}(\boldsymbol{r})$ is known, the capillary flow velocity, $\boldsymbol{v}(\boldsymbol{r})$ can be determined as has been suggested in detail by Deegan et al. ${ }^{3}$ At elevated substrate temperature, once the contact line gets pinned, the thickness of vertical liquid film reduces reasonably faster which results in much higher evaporation flux as well as bulk material transfer at the droplet periphery in a reasonably short duration. The strength of the capillary flow becomes reasonably high during such small period of time. It gets reduced significantly at the very end of the deposition process once most of the solutes get transferred towards the edge leading to a much flatter liquid film in the middle.

\section{References}

1. Li, Y., Lv, C., Li, Z., Quéré, D., Q. Zheng, Q. From coffee rings to coffee eyes. Soft Matter 2015, 11, 4669-4673.

2. Zhong, X.; Wu, C.; Duan, F. From enhancement to elimination of dual-ring pattern of nanoparticles from sessile droplets by heating the substrate. Appl. Therm. Eng. 2017, 115, 14181423.

3. Deegan, R. D.; Bakajin, O.; Dupont, T.F.; Huber, G.; Nagel, S. R.; Witten, T. A. Capillary flow as the cause of ring stains from dried liquid drops. Nature 1997, 389, 827-829 